This item was submitted to Loughborough's Research Repository by the author.

Items in Figshare are protected by copyright, with all rights reserved, unless otherwise indicated.

\title{
Evaluation of a torque-driven model of jumping for height
}

PLEASE CITE THE PUBLISHED VERSION

PUBLISHER

(C) Human Kinetics

VERSION

AM (Accepted Manuscript)

LICENCE

CC BY-NC-ND 4.0

REPOSITORY RECORD

King, Mark A., Cassie Wilson, and Maurice R. Yeadon. 2019. "Evaluation of a Torque-driven Model of Jumping for Height". figshare. https://hdl.handle.net/2134/6127. 
This item was submitted to Loughborough's Institutional Repository (https://dspace.lboro.ac.uk/) by the author and is made available under the following Creative Commons Licence conditions.

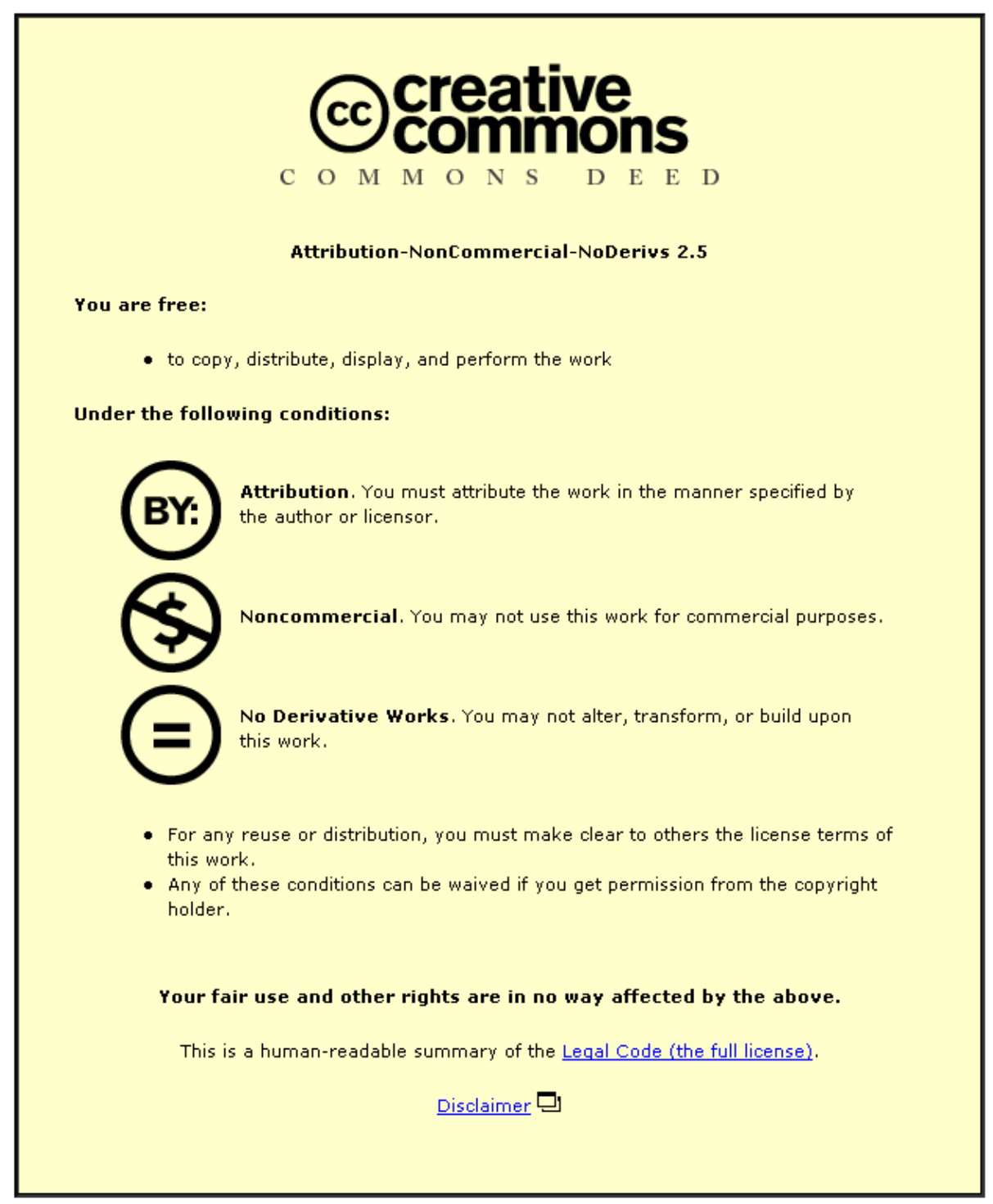

For the full text of this licence, please go to: http://creativecommons.org/licenses/by-nc-nd/2.5/ 


\title{
Evaluation of a torque-driven model of jumping for height
}

\author{
Mark A. King ${ }^{1}$, Cassie Wilson ${ }^{2}$ and Maurice R. Yeadon ${ }^{1}$ \\ ${ }^{1}$ School of Sport and Exercise Sciences, Loughborough University, Loughborough LE11 3TU, UK. \\ ${ }^{2}$ School of Sport, PE and Recreation, University of Wales Institute Cardiff, Cardiff, CF23 6XD, UK.
}

\begin{abstract}
This study used an optimisation procedure to evaluate an 8-segment torque-driven subject-specific computer simulation model of the takeoff phase in running jumps for height. Kinetic and kinematic data were obtained on a running jump performed by an elite male high jumper. Torque generator activation timings were varied to minimise the difference between simulation and performance in terms of kinematic and kinetic variables subject to constraints on the joint angles at takeoff to ensure that joints remained within their anatomical ranges of motion. A percentage difference of $6.6 \%$ between simulation and recorded performance was obtained. Maximising the height reached by the mass centre during the flight phase by varying torque generator activation timings resulted in a credible height increase of $90 \mathrm{~mm}$ compared with the matching simulation. These two results imply that the model is sufficiently complex and has appropriate strength parameters to give realistic simulations of running jumps for height.
\end{abstract}

Keywords: computer simulation, parameters, subject-specific

\section{Introduction}

While a number of studies have used computer simulation models to investigate vertical jumps from standing (Pandy et al., 1990; van Soest et al., 1994) the literature on the simulation of running jumps is rather sparse (Alexander, 1990; Hatze, 1981). The models of running jumps that have been developed have not been fully evaluated quantitatively from both a kinematic and kinetic perspective despite the fact that such a step is necessary in order to assess the results of simulations. Failure to carry out a quantitative evaluation carries the risk of not identifying gross modelling defects or simulation software errors.

Alexander (1990) used a simple two-segment model to determine optimum techniques in the long jump and the high jump and demonstrated that a suitable choice of model parameters lead to simulation results that were broadly comparable with competitive performances. Although sufficiently complex for its purpose of investigating general relationships, it is not feasible to use such a simple model to match a performance despite attempts by Linthorne and Kemble (1998) to do so.

At the other end of the model complexity scale, Hatze (1981) developed a 17-segment model to simulate the takeoff phase of the long jump. By varying the neural input to the model it was possible to match the centre of mass trajectory and the recorded ground reaction forces. Although this model was extremely complex in some respects (e.g. 55 muscle groups), the model was deficient in that no account was taken of segmental wobbling masses and these have been shown to affect ground reaction forces, joint torques and consequently the motion (Gruber et al., 1998; Pain and Challis, 2006). It appears that Hatze's result is anomalous in that it has not been replicated in the past 25 years and it would be prudent to include wobbling masses in models that are to be used for movements with an impact phase. In order to gain a realistic insight into the mechanics of performance a model needs to be sufficiently complex to represent the elements that have a substantial effect on performance.

Yeadon and King (2002) evaluated a subject-specific computer simulation model of tumbling by finding joint torque time histories that resulted in a simulation that closely matched a recorded performance. Good agreement was found between a double layout 
somersault performance and the matching simulation although the evaluation procedure was restricted to a matching of kinematic variables.

If a simulation model is to be used to investigate optimum technique in limiting movements such as jumps for maximum height it is crucial that the model has appropriate strength. Part of model evaluation should include an assessment of whether the model is sufficiently strong but not overly strong. In other words the model should be capable of reproducing a near maximal performance but should not be able to exceed this performance greatly. The purpose of this study was to evaluate a computer simulation model of the contact phase in running jumps for height using both kinetic and kinematic variables.

\section{Method}

An international male high jumper of height $1.89 \mathrm{~m}$ and mass $82 \mathrm{~kg}$, with a personal competition best of $2.31 \mathrm{~m}$ was used as the participant in the study. The athlete gave informed consent for the procedures, which were carried out in accordance with the protocol approved by Loughborough University Ethical Advisory Committee. Ninety-five anthropometric measurements were taken on the athlete and segmental inertia parameters were calculated using the geometric inertia model of Yeadon (1990b). Maximum voluntary torque time histories were measured at the ankle, knee and hip joints of the takeoff leg, the hip joint of the free leg, and the shoulder joint of the jumper using an active isovelocity dynamometer (Cybex NORM). The exercise protocol for each trial comprised two repetitions of a concentric-eccentric exercise at a preset crank angular velocity. The sequence of crank angular velocities of the concentric-eccentric trials was 50,100, 150, 200, 250, 300, 350, 400 and $450^{\circ} \mathrm{s}^{-1}$. A running jump in which the athlete took off from one leg with the aim of jumping as high as possible was recorded in a laboratory setting (Figure 1). Force data were collected during the contact phase of the trial using a Kistler force plate sampling at $800 \mathrm{~Hz}$. Two $50 \mathrm{~Hz}$ Sony digital Handycam VX1000 cameras and a NAC high speed HSV-400 video camera operating at $200 \mathrm{~Hz}$ were used to obtain displacement data (Wilson et al., 2006). The displacement data and the inertia data were then used to calculate the mass centre displacement and the orientation and configuration angles throughout the movement (Yeadon, 1990a) and these were fitted using quintic splines (Wood and Jennings, 1979) in order to calculate velocity estimates and the angular momentum about the mass centre (Yeadon, 1990c).

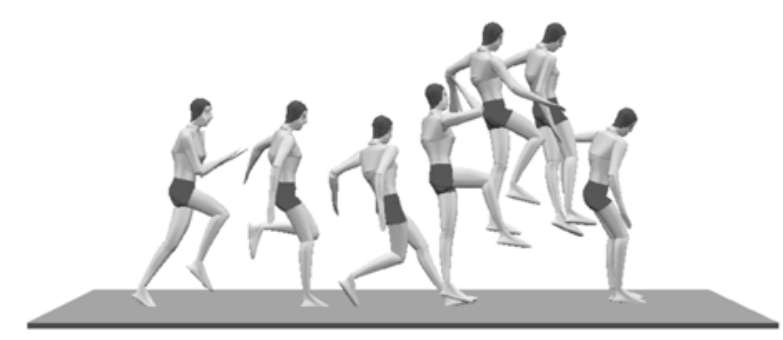

Figure 1. Graphics sequence of the running jump for height.

A planar eight-segment forward dynamics computer simulation model was developed for the foot contact phase in running jumps (Figure 2). The eight segments comprised foot, shank and thigh of the takeoff leg, thigh and shank+foot of the free leg, trunk+head, upper arm, and lower arm+hand (representing both arms). Torque generators, comprising rotational elastic and contractile elements in series, acted around five of the joints (ankle, knee and hip of the takeoff leg; hip of the free leg and shoulder) with extensors and flexors represented separately. The joint angle (external angle for the extensors) was expressed as the sum of a "contractile element angle" and an "elastic element angle" equivalent to the total length of a muscle-tendon complex. The remaining two joints in the model (elbow and free knee) were 
angle-driven using data from the recorded jump. Movement of the soft tissue in the takeoff leg and trunk was modelled using (rigid) wobbling masses connected by visco-elastic elements to fixed linked masses representing the bones of the shank, thigh and trunk segments. The foot-ground interface was modelled in a similar way using damped springs with horizontal and vertical elements situated between the ground and the heel, and the ground and the toe (Wilson et al., 2006).

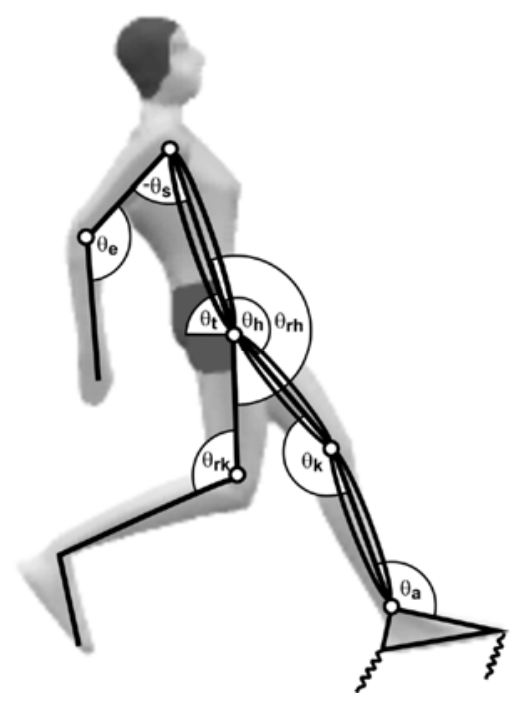

Figure 2. The eight-segment simulation model of the takeoff in jumping. Wobbling masses are situated within the shank, thigh and trunk segments and springs situated at the heel and toe represent the foot-ground interface.

Subject-specific inertia and visco-elastic parameters were calculated for the simulation model from measurements taken on the jumper, from data in the literature and from simulations using an angle-driven model of jumping as described in Wilson et al. (2006). Inertia parameters for the fixed and wobbling masses were calculated from the subjectspecific segmental inertia parameters using ratios of wobbling mass to fixed mass based on data from Clarys and Marfell-Jones (1986) and modelling the fixed mass in a segment as a uniform cylinder of known length and with a density value of $1.1 \mathrm{~kg} .1^{-1}$ (Dempster, 1955). Visco-elastic parameters for the attachment of wobbling masses to fixed links and for the foot-ground interface were determined using an eight-segment angle-driven simulation model to match two running jumps by the subject. The visco-elastic parameters were varied using Simulated Annealing optimisation (Corana et al., 1987) until parameter values were found that resulted in simulations that closely matched the two actual performances in terms of trunk orientation at takeoff, joint angles at takeoff, time of contact, linear and angular momenta at takeoff and horizontal and vertical ground reaction force time histories.

Nine parameters were required for each torque generator to define the maximum voluntary torque as a function of contractile element angle and angular velocity. Seven of the parameters defined maximum voluntary torque at a joint as a function of contractile element angular velocity (Yeadon et al., 2006). Four of these parameters defined the tetanic torque / angular velocity profile while the remaining three parameters defined the differential activation / angular velocity profile for maximal voluntary activations. This three-parameter function allowed the maximum voluntary activation to rise as a monotonic function of angular velocity from a depressed level $\mathrm{a}_{\min }$ in the eccentric mode to full activation in concentric mode (Westing et al., 1990, 1991). Two additional parameters defined maximum torque as a quadratic function of contractile element angle (Table 1). The nine parameters for each torque generator were determined by matching the torque function values to the measured 
isovelocity torques using Simulated Annealing optimisation. A stiffness parameter for a rotational series elastic element was determined from published data (Pierrynowski, 1995; Jacobs et al., 1996) using a 5\% estimate of maximum stretch for the series elastic element at maximum isometric torque (de Zee and Voigt, 2001; Muramatsu et al., 2001).

The torque at a joint was calculated as the product of the maximum voluntary torque (given by the nine-parameter function) and an activation level. The time history of the activation of each torque generator ranged from zero up to a possible maximum value of 1 and was allowed to rise and then fall for the agonists and to fall and then rise for the antagonists as specified by a specific activation profile. Two different profile types were used to represent the activation time histories of the agonist (ankle, knee and hip extensor; shoulder and free hip flexor) and antagonist (ankle, knee and hip flexor; shoulder and free hip extensor) torque generators. Six parameters were needed to specify the activation time histories of each agonist torque generator (Figure 3a). During the first half of the profile the activation level used was the higher of the initial activation level $a_{i}$ and the activation level specified by the first quintic function. In the second half of the profile the activation level used was the lower of the upper activation level $\mathrm{a}_{\mathrm{u}}$ and the activation level specified by the second quintic function. Each quintic function was defined by start time, end time, start value and end value (Yeadon and Hiley, 2000). Five parameters were needed to specify the activation time histories of each antagonist torque generator (Figure 3b). During the first half of the profile the activation level used was the lower of $a_{i}$ and the activation level specified by the first quintic function. In the second half of the profile the activation level was specified by the second quintic function which was assumed to have an end value of 1 . This reduced the number of parameters from six to five and hence simplified the optimisations.

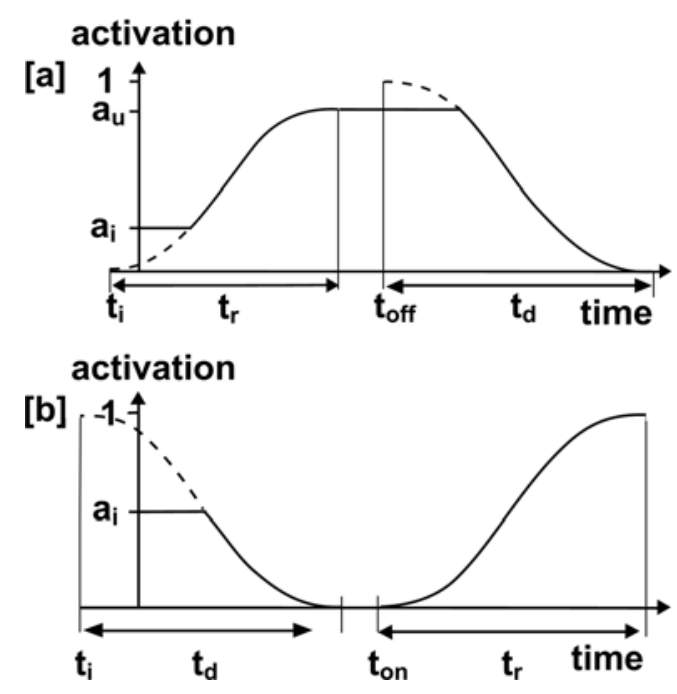

Figure 3. (a) Six-parameter profile for the agonist torque generators. $a_{i}$ is the initial activation level, $t_{i}$ corresponds to the time the first quintic function ramps up from zero activation, $t_{r}$ is the time taken for the first quintic function to reach the upper activation level $a_{u} \cdot t_{\text {off }}$ is the time the second quintic function starts to ramp down from an activation level of 1.0 and $t_{d}$ is the time taken for the activation level to fall to zero. (b) Similar five-parameter profile for the antagonist torque generators. $a_{i}$ is the initial activation level, $t_{i}$ corresponds to the time the first quintic function starts to ramp down from an activation level of 1.0, $t_{d}$ is the time taken for the first quintic function to reach zero activation. $t_{\text {on }}$ corresponds to the time the second quintic function ramps up from zero activation, $t_{r}$ is the time taken for the second quintic function to reach an activation level of 1.0.

The FORTRAN code implementing the model was generated using the Autolev software package (www.autolev.com) based on Kane's equations (Kane and Levinson, 1985). 
Input to the simulation model comprised mass centre velocity, the angle and angular velocity at each joint at touchdown, the joint angle time histories of the free knee and elbow, and the activation profiles for each of the torque generators. The output from the model comprised time histories of the whole body angular momentum about the mass centre, the mass centre velocity, displacements of the wobbling masses relative to the fixed links, compression of the foot-ground interface, and the orientation and angular velocity of each segment during the contact phase. The kinematics at takeoff were used as the initial conditions for an 11-segment model of aerial movement (Yeadon et al., 1990). The aerial model required time histories of the configuration throughout flight. Over the first $100 \mathrm{~ms}$ of the flight phase the configuration at takeoff of the simulation was merged into the configuration used in the performance using a quintic function (Yeadon and Hiley, 2000) to join the joint angle values at takeoff with the angles $100 \mathrm{~ms}$ into flight.

The first stage in evaluating the simulation model was to find a simulation that closely matched the performance. The inertia, visco-elastic and torque parameter values remained fixed at the calculated values. The initial conditions for the simulation were estimated from the image analysis. The mass centre velocity was fixed at the value estimated from the image analysis as it was considered sufficiently accurate. The five initial joint angular velocities and trunk segment angular velocity were allowed to vary by $\pm 50^{\circ} \mathrm{s}^{-1}$ as these were not considered to be so accurate (Yeadon and King, 2002). The Simulated Annealing algorithm (Corana et al, 1987) varied the 55 torque generator activation parameters (5 joints and 11 parameters per joint, Figure 3) and the six initial segment angular velocities in order to minimise a cost function which consisted of six components to assess how well the simulated and recorded performances matched. Component (1) was the absolute difference in the trunk orientation at takeoff (measured in degrees); component (2) was the RMS difference in the joint angles at takeoff and the minimum angles reached at the ankle, knee and hip (measured in degrees); component (3) was the percentage absolute difference in the time of contact; component (4) was the percentage RMS difference in the horizontal and vertical linear momentum at takeoff; component (5) was the percentage absolute difference in the angular momentum at takeoff; component (6) was the overall RMS difference in the time histories of the horizontal and vertical ground reaction forces during the takeoff phase as a percentage of peak force. The whole body angular momentum at takeoff was small so the weighting of component (5) was adjusted so that a $1 \%$ error in the angular momentum at takeoff was equivalent to $1^{\circ}$ error in rotation on landing after the flight phase. The overall RMS difference expressed as a percentage was then calculated from the six components with all components equally weighted since differences in degrees and percentages were considered to give comparable measures (Yeadon and King, 2002).

Anatomical constraints on the knee and ankle joints during takeoff and the first part of flight were used to ensure that simulations were realistic. The knee and ankle joint angles (Figure 2) were not allowed to exceed $180^{\circ}$ and $160^{\circ}$ respectively either at takeoff or during the first $100 \mathrm{~ms}$ of the flight phase assuming constant angular acceleration. If a simulation resulted in any of the constraints being violated, penalties were added to the score for the simulation. No penalties were incurred in the final matching solution.

A comparison was made between the matching simulation and recorded performance in terms of the time histories of joint angles, trunk orientation, mass centre displacements and ground reaction forces. In addition the time histories of the joint torques used in the matching simulation were compared with estimates obtained from inverse dynamics analysis of the recorded performance (van den Bogert and de Koning, 1996).

The second stage in evaluating the simulation model was to maximise the height reached by the mass centre in a simulation using the initial conditions from the matching simulation and varying the 55 torque generator activation parameters using Simulated 
Annealing. The score used in the optimisation was equal to the peak height reached by the mass centre in flight minus penalties associated with violation of anatomical constraints at the knee and ankle joints. No penalties were incurred in the optimised simulation. The height of the optimised jump was then compared with the jump height of the matching simulation to check that the difference was not unrealistically large.

To ensure that the Simulated Annealing algorithm found the global optimum in each optimisation, additional optimisations were run with different initial parameter estimates and with the parameters in a different order. The additional optimisations produced no improvement in the global optima obtained in the two optimisations.

\section{Results}

Subject-specific parameters were determined for the high jumper who took part in this study. The inertia and visco-elastic parameters for the model have been presented in a previous paper (Wilson et al., 2006). The torque parameters at each joint were defined using a nine-parameter function with one additional parameter for the stiffness of the series elastic component (Table 1). The initial conditions for the matching simulation were based upon the kinematic data calculated from the image analysis of the performance (Table 2).

Table 1. Ten parameters for each torque generator in the simulation model

\begin{tabular}{|c|c|c|c|c|c|c|c|}
\hline & $\begin{array}{c}\text { knee } \\
\text { ext }\end{array}$ & $\begin{array}{c}\text { knee } \\
\text { flex }\end{array}$ & $\begin{array}{c}\text { hip } \\
\text { ext }\end{array}$ & $\begin{array}{c}\text { hip } \\
\text { flex }\end{array}$ & $\begin{array}{c}\text { shld } \\
\text { flex }\end{array}$ & $\begin{array}{c}\text { ank } \\
\text { plant }\end{array}$ & $\begin{array}{c}\text { ank } \\
\text { dorsi }\end{array}$ \\
\hline $\mathrm{T}_{\max }[\mathrm{Nm}]$ & 491 & 322 & 719 & 433 & 286 & 424 & 96 \\
\hline $\mathrm{T}_{0}[\mathrm{Nm}]$ & 328 & 215 & 480 & 289 & 190 & 283 & 65 \\
\hline$\omega_{\max }\left[\mathrm{rad} . \mathrm{s}^{-1}\right]$ & 13.4 & 15.0 & 14.2 & 24.9 & 28.8 & 15.7 & 15.7 \\
\hline$\omega_{\mathrm{c}}\left[\mathrm{rad} . \mathrm{s}^{-1}\right]$ & 21.2 & 16.5 & 3.2 & 14.0 & 4.5 & 15.3 & 15.3 \\
\hline $\mathrm{a}_{\min }$ & 0.56 & 0.58 & 0.46 & 0.69 & 0.24 & 0.57 & 0.57 \\
\hline $\mathrm{m}$ & 0.49 & 0.43 & 0.29 & 5.02 & 0.00 & 0.46 & 0.46 \\
\hline$\omega_{1}\left[\mathrm{rad} . \mathrm{s}^{-1}\right]$ & 1.1 & 0.59 & 0.45 & 6.00 & 0.00 & 0.86 & 0.86 \\
\hline$\theta_{\text {opt }}[\mathrm{rad}]$ & 2.0 & 3.6 & 1.6 & 3.6 & 0.5 & 1.5 & 1.9 \\
\hline $\mathrm{k}_{2}$ & 0.53 & 0.08 & 0.27 & 0.33 & 0.09 & 0.43 & 0.55 \\
\hline $\mathrm{K}\left[\mathrm{Nm} . \mathrm{rad}^{-1}\right]$ & 857 & 340 & 5092 & 496 & 3000 & 767 & 223 \\
\hline
\end{tabular}

Note: Maximum voluntary torque exerted at a joint $=\mathrm{T}_{(7)}\left(1-\mathrm{k}_{2}\left(\theta_{\mathrm{ce}}-\theta_{\text {opt }}\right)^{2}\right)=\mathrm{K} \theta_{\text {see }}$ where $\mathrm{T}_{(7)}$ is the maximum voluntary torque for a given contractile element angular velocity (See Yeadon et al. (2006) for full details), $\theta_{\mathrm{ce}}=$ contractile element angle, $\theta_{\text {see }}=$ series elastic element angle. The function $\mathrm{T}_{(7)}$ has parameters: maximum eccentric torque $\mathrm{T}_{\max }$, isometric torque $\mathrm{T}_{0}$, maximum contractile element angular velocity $\omega_{\max }$, asymptote velocity $\omega_{\mathrm{c}}$ of the concentric hyperbola, depressed activation level $\mathrm{a}_{\min }$ in the eccentric phase, slope parameter $1 / \mathrm{m}$ of the differential activation function, and inflection velocity $\omega_{1}$ of the differential activation function. Values for shoulder torque are for the double arm in the model. 
Table 2. Initial conditions for the matching simulation at touchdown with the force plate

\begin{tabular}{|c|c|c|c|}
\hline variable & $\begin{array}{c}\text { matching } \\
\text { simulation }\end{array}$ & variable & $\begin{array}{c}\text { matching } \\
\text { simulation }\end{array}$ \\
\hline $\mathrm{v}_{\mathrm{cmx}}$ & $4.4 \mathrm{~ms}^{-1}$ & $\mathrm{v}_{\mathrm{cmy}}$ & $-0.85 \mathrm{~ms}^{-1}$ \\
\hline$\theta_{\mathrm{a}}$ & $135^{\circ}$ & $\dot{\theta}_{\mathrm{a}}$ & $-12^{\circ} \mathrm{s}^{-1}$ \\
\hline$\theta_{\mathrm{k}}$ & $157^{\circ}$ & $\dot{\theta}_{\mathrm{k}}$ & $-287^{\circ} \mathrm{s}^{-1}$ \\
\hline$\theta_{\mathrm{h}}$ & $149^{\circ}$ & $\dot{\theta}_{\mathrm{h}}$ & $147^{\circ} \mathrm{s}^{-1}$ \\
\hline$\theta_{\mathrm{s}}$ & $-55^{\circ}$ & $\dot{\theta}_{\mathrm{S}}$ & $931^{\circ} \mathrm{s}^{-1}$ \\
\hline$\theta_{\mathrm{e}}$ & $134^{\circ}$ & $\dot{\theta}_{\mathrm{e}}$ & $-75^{\circ} \mathrm{s}^{-1}$ \\
\hline$\theta_{\mathrm{rh}}$ & $185^{\circ}$ & $\dot{\theta}_{\mathrm{rh}}$ & $-285^{\circ} \mathrm{s}^{-1}$ \\
\hline$\theta_{\mathrm{rk}}$ & $108^{\circ}$ & $\dot{\theta}_{\mathrm{rk}}$ & $-584^{\circ} \mathrm{s}^{-1}$ \\
\hline$\theta_{\mathrm{t}}$ & $78^{\circ}$ & $\dot{\theta}_{\mathrm{t}}$ & $-35^{\circ} \mathrm{s}^{-1}$ \\
\hline
\end{tabular}

Note: See Figure 2 for angle definitions, $\mathrm{v}_{\mathrm{cmx}}$ and $\mathrm{v}_{\mathrm{cmy}}$ are the horizontal and vertical velocities of the mass centre at touchdown.

Close agreement was found between the performance and the matching simulation with a difference score of $6.6 \%$ (components $1-6$ had individual scores of: $0.8^{\circ}, 5.0^{\circ}, 0.0 \%, 2.4 \%$, $0.7 \%$, and $15.2 \%$ respectively). In general the differences between the simulation and performance in terms of the kinematic and kinetic variables used in the difference score were relatively small (Table 3, Figure 4). This resulted in a close match between the simulation and the performance during the flight phase with peak height reached by the mass centre being only $0.01 \mathrm{~m}$ different $(1.82 \mathrm{~m}$ for the simulation compared to $1.81 \mathrm{~m}$ for the performance). The ankle and knee joint net torques in the matching simulation were comparable with the inverse dynamics estimates with differences in peak torque of $+11 \%$ and $-12 \%$ (Figure 5). The correspondence between the peak hip torque values was less close ($44 \%$ ). The upper activation levels for the extensors were 0.91 at the ankle, 1.00 at the knee, and 0.97 at the hip suggesting that the measured hip strength estimate might be too low although the inverse dynamics peak hip torque estimate might be too high due to artifacts in the inverse dynamics estimates (van den Bogert and de Koning, 1996). 
Table 3. Comparison of kinematic variables at takeoff for the performance, matching simulation and optimisation for height

\begin{tabular}{|l|c|c|c|}
\hline \multicolumn{1}{|c|}{ variable } & performance & $\begin{array}{c}\text { matching } \\
\text { simulation }\end{array}$ & $\begin{array}{c}\text { optimisation } \\
\text { for height }\end{array}$ \\
\hline $\mathrm{v}_{\mathrm{cmx}}\left(\mathrm{ms}^{-1}\right)$ & 1.91 & 1.94 & 1.93 \\
\hline $\mathrm{v}_{\mathrm{cmy}}\left(\mathrm{ms}^{-1}\right)$ & 3.31 & 3.25 & 3.49 \\
\hline $\mathrm{h}_{\mathrm{g}}\left(\mathrm{kgm}^{2} \cdot \mathrm{rads}^{-1}\right)$ & 4.9 & 5.1 & 5.1 \\
\hline time of contact & $0.205 \mathrm{~s}$ & $0.205 \mathrm{~s}$ & $0.197 \mathrm{~s}$ \\
\hline orientation $\theta_{\mathrm{t}}$ & $86^{\circ}$ & $85^{\circ}$ & $85^{\circ}$ \\
\hline ankle angle $\theta_{\mathrm{a}}$ & $140^{\circ}$ & $153^{\circ}$ & $159^{\circ}$ \\
\hline knee angle $\theta_{\mathrm{k}}$ & $167^{\circ}$ & $166^{\circ}$ & $170^{\circ}$ \\
\hline hip angle $\theta_{\mathrm{h}}$ & $179^{\circ}$ & $173^{\circ}$ & $173^{\circ}$ \\
\hline sh. angle $\theta_{\mathrm{s}}$ & $105^{\circ}$ & $105^{\circ}$ & $105^{\circ}$ \\
\hline r. hip angle $\theta_{\mathrm{rh}}$ & $94^{\circ}$ & $92^{\circ}$ & $92^{\circ}$ \\
\hline
\end{tabular}

Note: $\mathrm{v}_{\mathrm{cmx}}$ and $\mathrm{v}_{\mathrm{cmy}}$ are the horizontal and vertical velocities of the mass centre at takeoff, $\mathrm{h}_{\mathrm{g}}$ is the angular momentum about a transverse axis through the mass centre and see Figure 2 for the angle definitions.
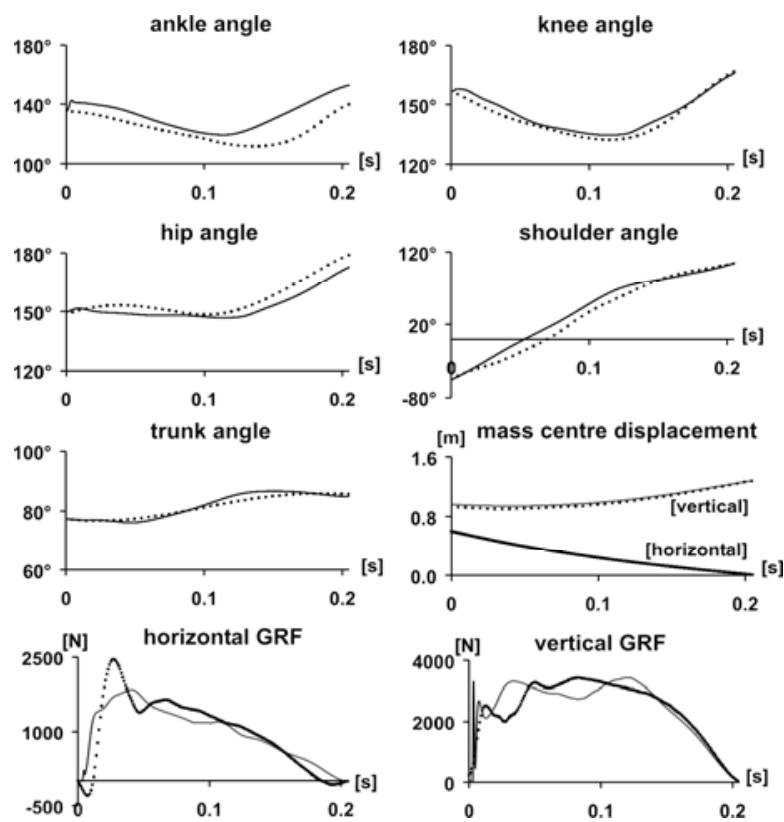

Figure 4. Comparison of key kinematic and kinetic variables during the takeoff phase for the jump for height. The solid line represents the matching simulation and the dotted line represents the performance. 

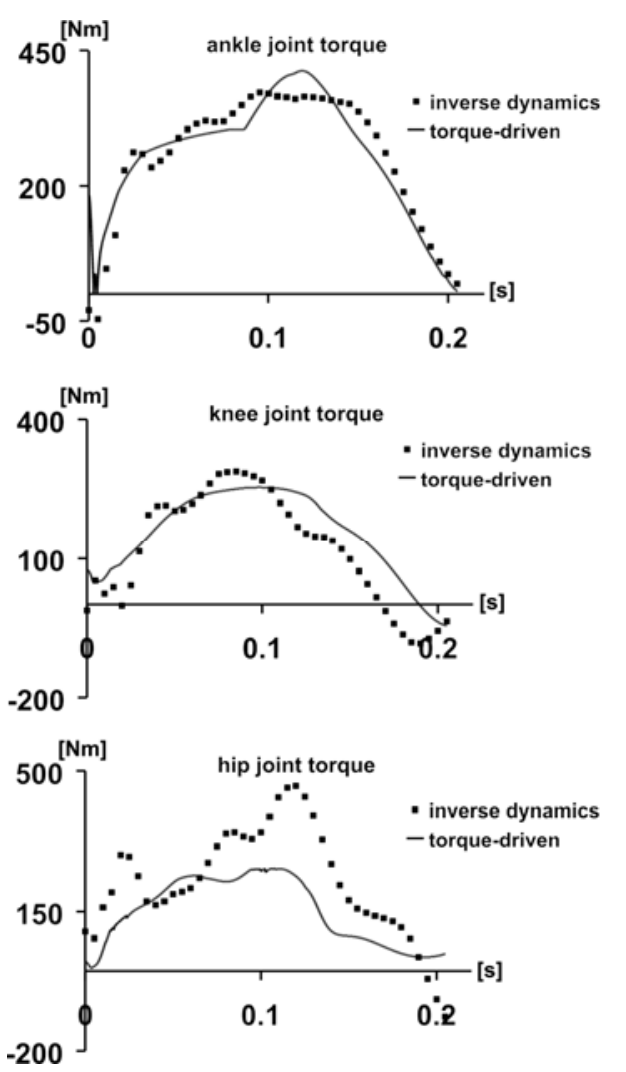

Figure 5. Comparison of torques used in the matching simulation (solid line) with torques calculated from inverse dynamics (discrete points).

The model was subsequently used to maximise the height reached by the mass centre in a jump for height. By keeping the same initial conditions as in the matching simulation and simply varying the torque generator activation parameters, the height reached by the mass centre increased by $0.09 \mathrm{~m}$ from $1.82 \mathrm{~m}$ to $1.91 \mathrm{~m}$ (Figure 6). The matching simulation and optimised simulation used similar activation profiles (Figure 7). In both simulations the extensors rose from an initial level to a maximal or near maximal level before dropping towards the end of the takeoff while the flexors dropped down to zero activation during the first part of the takeoff phase and then came on towards the end of the takeoff phase.

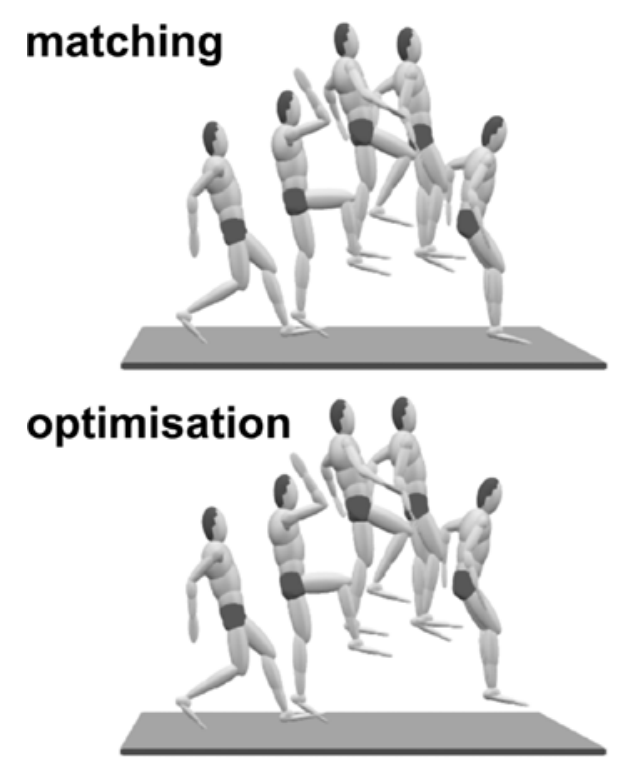

Figure 6. Comparison of matching simulation and optimised simulation for height. 


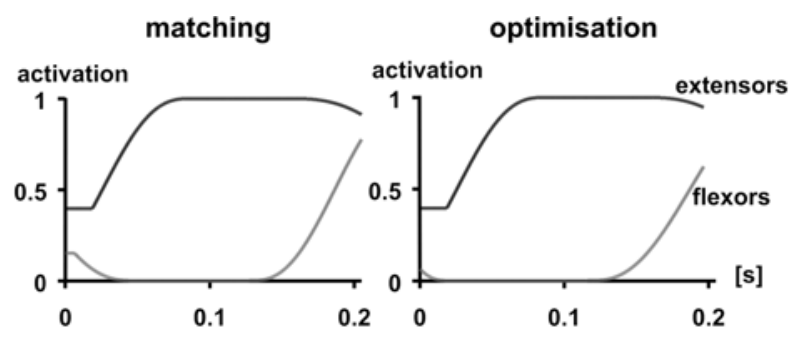

Figure 7. Activation profile of the knee extensors and flexors for the matching simulation and the optimised simulation for height.

\section{Discussion}

This paper has described a method of evaluating a torque-driven model that can be used to simulate the contact phase of running jumps for height. The model has been evaluated quantitatively by comparing both kinetic and kinematic variables from a simulation with corresponding variables from a recorded performance. Close agreement between performance and simulation was achieved with an overall difference of $6.6 \%$, resulting in a difference of only $0.01 \mathrm{~m}$ in jump height. Requiring the model to match both the kinematics and kinetics during the takeoff phase was challenging and was an improvement on previous evaluations of models of running jumps where only the kinematics (Yeadon and King, 2002) or kinetics (Hatze, 1981) were matched. Despite the fact that only minimum joint angles and angles at takeoff (rather than time histories) were matched, there was close agreement between the time histories of the joint angles, trunk orientation and mass centre displacement (Figure 4). Additionally there was reasonable agreement between the simulation and performance values of the ground reaction forces and joint torques despite using simplified joint torque activation profiles (Figures 4,5 ). The matching procedure shows that there exists a simplified activation time history at each joint that produces a simulation that closely matches the recorded performance.

The performance was recorded after the competitive season had ended and so the athlete might not have been jumping at his full potential. As a consequence it is to be expected that the actual jump height achieved would be slightly below the theoretical optimum. Indeed the matching simulation was only $0.09 \mathrm{~m}$ below the maximum jump height of $1.91 \mathrm{~m}$ reached by the optimised simulation. This indicates that the subject-specific torque parameters in the model are of the correct magnitude since too strong a model would have resulted in too high an optimum and too weak a model would not have been able to match the recorded performance. Comparing the optimised and matching simulations (Table 3, Figures 6 and 7) shows that the techniques used were similar with the ankle and knee more extended at takeoff in the optimised solution.

The fact that many tracking studies can match the kinematics closely without the use of subject-specific parameters is most likely a consequence in cycling (Thelen et al., 2003) of the tracked movement having a small number of degrees of freedom and being submaximal whereas in walking (Anderson and Pandy, 2001) tracking is facilitated since the motion can be largely accounted for using a passive model (Collins et al., 2005). In contrast the movement in the present paper is an example of a maximal (limiting) activity with few constraints. In this dynamic movement the body experiences near maximal loading in the eccentric phase before producing a maximal effort in the concentric phase. In order to perform at the correct level it is essential that the model should have appropriate strength and inertia parameters. While it may be possible to match recorded data with a model that is too strong, this will not be possible if the model is too weak. Additionally a model that is too strong is likely to produce an optimum performance that is unachievable in practice. 
The process of evaluation establishes the level of accuracy that might be expected from model simulations and should be borne in mind when reaching conclusions using the results of simulations. To be considered an accurate representation the difference between simulation and performance should be less than 10\%. This level of accuracy cannot be achieved for maximal movements using simple one-segment or two-segment models that typically give performance measures within a factor of two of actual performance. While such simple models may be sufficient to illustrate some general principles (e.g. Alexander, 1990) they are inadequate for quantitative analyses of optimisation and performance contributions or for sensitivity studies. While it may be argued that validating all aspects of a model is a theoretical impossibility (Oreskes et al., 1994) this should not be taken as an excuse to omit an assessment of model accuracy. The process of modelling should be an interactive one that iterates to an appropriate solution but without some quantitative assessment of model performance such iteration of model development cannot occur. If a model has not been shown to reproduce performance to within $10 \%$ the danger is that some aspect has not been modelled adequately or has not been modelled at all. As a consequence results obtained from the model may be misleading.

It has been argued that the simplest possible model should be used that will represent the essential features of the human system (Sprigings and Miller, 2004). This might be interpreted by a choice of the simple two-segment jumping model of Alexander (1990). Although it is possible to tune this model to jump as high as international competitors $(2.35$ $\mathrm{m})$ this is only achievable using a knee extensor torque more than twice that of an elite athlete (Wilson, 2003). If the aim is to obtain accurate simulations using realistic parameter values then those elements that are known to have a measurable effect on performance should be regarded as essential features.

It has been shown that appreciable power is developed in vertical jumping about the hip, knee and ankle joints (Bobbert et al., 1986) so that it is necessary to include segments representing trunk+head, and thigh, shank and foot of the takeoff leg. Dapena (1999) has shown the importance of the movement of the free limbs during the contact phase of jumps for height and so the inclusion of segments representing the free leg and the arms is also necessary. Pain and Challis (2006) have shown that for impacts such as drop landings it is necessary to include wobbling masses within the trunk, thigh and shank segments since these affect the joint torques required for a given movement. This indicates that it is necessary to include wobbling masses within the segments of the plant leg since the bony structures are constrained by the ground contact whereas the free leg segments may be modelled as rigid bodies since they have no such constraint. Visco-elastic elements are required to represent the foot-ground interface as described by Pain and Challis (2001). Since flexors are used to prevent hyperextension of the joints, both extensor and flexor torque generators are needed at the five torque-driven joints. Activation time histories are needed with sufficient flexibility to allow agonist torques to rise and then fall and antagonist torques to fall and then rise.

It may be argued therefore that the inclusion of the above elements in the model is necessary. Omitting any of the above features might be expected to result in a degraded performance and a decreased ability to match the recorded performance. For example, if the ability to exert torque at the ankle is removed from the model and a new matching simulation is determined by optimisation, the difference score increases from $6.6 \%$ to $31.9 \%$ and the jump height drops from $1.82 \mathrm{~m}$ to $1.24 \mathrm{~m}$. If the stiffness and damping of the wobbling masses are increased 100 times to approximate rigid segments and the foot-ground interface parameters are re-optimised, the difference score increases by more than a quarter from $6.6 \%$ to $8.3 \%$. While this is a more modest deterioration than in the previous example, it comes at a cost of allowing the foot-ground interface parameters to compensate for the omission of 
wobbling masses. This affects the foot displacements during contact, the ground reaction forces, and the joint torques.

The knee joint and elbow joint of the free limbs were driven kinematically since this ensures that the configurations used in all simulations are representative of an elite performance and keeps the number of variables in a simulation to a minimum. As these joints are near the end of the link system their influence on performance is limited. Since the model was able to produce a close match to the actual performance it may be argued that the model is sufficiently complex and is appropriate for simulating running jumps.

A simplification of the model is its limitation to planar movements as this does not allow the rotation about a frontal axis that typically occurs in the Fosbury flop style of high jumping. However since the body is close to vertical during a competitive high jump takeoff it may be expected that a planar jump would rise to a similar height for similar approach characteristics. Other limitations of the model revolve around the simplifications that have been made in order to allow subject-specific parameters to be determined for the model. In particular, increases in model complexity such as a multi-segment foot, more complex joint torque activations, or biarticular muscle representations may improve the match between simulation and performance, but in this study the level of agreement obtained (mean difference less than 7\%) was deemed to be sufficient. The ground reaction forces (Figure 4) proved to be the most difficult to match. Possibilities for improving this match are a multisegment foot, more complex activation profiles, and wobbling masses with elastic characteristics that vary with muscle activation. The inclusion of a multi-segment foot might also improve the matching of the later phase of the ankle angle time history. A closer matching of the other joint angles during the takeoff phase (Figure 4) would have required more complex activation profiles and therefore more parameters to be varied. The present scheme of defining activation profiles using five or six parameters was more complex than those used by Sprigings and Miller (2004) in diving takeoffs (two parameters) and by King and Yeadon (2005) in vaulting (four parameters) but comparable with that used by Cheng and Hubbard (2004) for springboard diving (10 parameters but no antagonists).

Additional parameters could be used to represent the action of biarticular muscles since these may have an important role in jumping (Bobbert and van Ingen Schenau, 1988). The magnitude of such a role, however, appears to be relatively small (Pandy and Zajac, 1991; van Soest et al., 1993) and contributions from such muscles are implicitly included in the joint torques although the dependence of the joint torque on motion at the second joint is not represented. For this study it was decided that varying as few parameters as possible while still being able to match the performance relatively well was better than having more parameters and achieving a closer match with the concurrent danger of over-fitting the performance data.

Incorporating anatomical constraints within the matching and optimisation procedure resulted in simulations with realistic joint angles and angular velocities at takeoff. Further work is needed in the future to establish appropriate anatomical constraints more precisely since the constraints imposed may affect the results obtained from optimisations. The activation profiles used resulted in a close match of the performance and so the profiles used are not too simple for simulating running jumps. Even with simple activation profiles, 55 activation parameters were needed and so in the future it would be desirable to find ways to reduce the number of parameters to be varied in an optimisation. In addition further work is needed to consider how robust optimum solutions are to perturbations of the approach and activation timings, as it is likely that elite performers develop techniques that are relatively insensitive to perturbations.

The weakness of many simulation models is that the level of accuracy is unknown (Yeadon and Challis, 1994) whereas carrying out an evaluation permits the model accuracy to 
be established and also helps in identifying modelling deficiencies. It seems to be quite common and apparently acceptable for journals to publish optimisation studies using simulation models giving quantitative results on performance (distances, velocities) and technique (joint torques, activations) without accompanying quantitative assessments of model accuracy (e.g. Ashby and Delp, 2006; Nagano and Gerritsen, 2001; Sprigings and Miller, 2004). This is not to suggest that the models in these studies are deficient - only that they have not been demonstrated to be "fit for purpose". If studies are to be considered to be "scientific", however, journals should require models to be evaluated and should consider papers on model evaluation potentially worthy of publication.

In this study a model was tuned to an individual athlete by using subject-specific parameters so that a quantitative comparison between simulation and performance could be made. The close matching of the simulation with performance indicated that the model complexity (although minimal) was sufficient to produce an accurate simulation of a recorded performance. The close matching also showed that the torque generators were sufficiently strong. The fact that the optimisation produced only a small increase in jump height above the matching simulation indicated that the torque generators were not too strong.

The optimisation in this study allowed activation timings to vary in order to produce a jump of maximal height. In the future other parameters such as mass centre velocity, leg plant angle, and knee angle at touchdown will be also be allowed to vary when optimising jump height. In addition the model will be used to assess the influence of strength gains on optimum jumping performance and the influence of movement constraints on optimum jumping technique. The procedures described in this study have wide applicability to the evaluation of simulation models of maximal human movement.

\section{References}

Alexander, R.McN., 1990. Optimum take-off techniques for high and long jumps. Philosophical Transactions of The Royal Society B329, 3-10.

Anderson, F.C. and Pandy, M.G. 2001. Dynamic optimization of human walking. Journal of Biomechanical Engineering 123, 381-390.

Ashby, B.M., Delp, S.L., 2006. Optimal control simulations reveal mechanisms by which arm movement improves standing long jump performance. Journal of Biomechanics 39, 1726-1734.

Bobbert, M.F., Ingen Schenau, G.J. van, 1988. Coordination in vertical jumping. Journal of Biomechanics 21, 249-262.

Bobbert, M.F., Mackay, M., Schinkelshoek, D., Huijing, P., Ingen Schenau, G.J. van, 1986. Biomechanical analysis of drop and countermovement jumps. European Journal of Applied Physiology 54, 566-573.

van den Bogert, A.J., de Koning, J.J., 1996. On optimal filtering for inverse dynamics. In Proceedings of the IXth Biennial Conference of the Canadian Society for Biomechanics, pp. 214-215. Vancouver: Simon Fraser University.

Cheng, K. B., Hubbard, M., 2004. Optimal jumping strategies from compliant surfaces: a simple model of springboard standing jumps. Human Movement Science 23, 35-48.

Clarys, J.P., Marfell-Jones, M.J., 1986. Anthropometric prediction of component tissue masses in the minor limb segments of the human body. Human Biology 58, 761-769.

Collins, S., Ruina, A., Tedrake, R., Wisse M. 2005. Efficient bipedal robots based on passive-dynamic walkers. Science 307, 1082-1085. 
Corana, A., Marchesi, M., Martini, C., Ridella, S., 1987. Minimising multimodal functions of continuous variables with the "simulated annealing" algorithm. ACM Transactions on Mathematical Software 13, 262-280.

Dapena, J., 1999. A biomechanical explanation of the effect of arm actions on the vertical velocity of a standing jump. In International Society of Biomechanics XVIIth Congress Book of Abstracts, p. 100. University of Calgary.

De Zee, M., Voigt, M., 2001. Moment dependency of the series elastic stiffness in the human plantar flexors measured in vivo. Journal of Biomechanics 34, 1399-1406.

Dempster, W.T., 1955. Space requirements of the seated operator. WADC-TR 55-159. Dayton, OH: Wright-Patterson Air Force Base.

Gruber, K., Ruder, H., Denoth, J., Schneider, K., 1998. A comparative study of impact dynamics: wobbling mass model versus rigid body models. Journal of Biomechanics 31, 439-444.

Hatze, H., 1981. A comprehensive model for human motion simulation and its application to the take-off phase of the long jump. Journal of Biomechanics 14, 135-142.

Jacobs, R., Bobbert, M.F., van Ingen Schenau, G.J., 1996. Mechanical output from individual muscles during explosive leg extensions: The role of biarticular muscles. Journal of Biomechanics 29, 513-523.

Kane, T.R., Levinson, D.A., 1985. Dynamics: Theory and applications. New York: McGraw-Hill Book Company.

King, M.A. and Yeadon, M.R. 2005. Factors influencing performance in the hecht vault and implications for modelling. Journal of Biomechanics 38, 145-151.

Linthorne, N.P., Kemble, B.A., 1998. Take-off technique in the high jump. In Proceedings of the XVI International Symposium on Biomechanics in Sport, Konstanz, Germany (pp. 356-359).

Muramatsu, T., Muraoka, T., Takeshita, D., Kawakami, Y., Hirano, Y., Fukunaga, T., 2001. Mechanical properties of tendon and aponeurosis of human gastrocnemius muscle in vivo. Journal of Applied Physiology 90, 1671-1678.

Nagano, A., Gerritsen, G.M., 2001. Effects of neuromuscular strength training on vertical jumping performance - A computer simulation study. Journal of Applied Biomechanics 17, 113-128.

Oreskes, N., Shrader-Frechette, K., Belitz, K., 1994. Verification, validation and confirmation of numerical models in the earth sciences. Science 263, 641-646.

Pain, M.T.G., Challis, J.H., 2001. The role of the heel pad and shank soft tissue during impacts: a further resolution of a paradox. Journal of Biomechanics 34, 327-333.

Pain, M.T.G. and Challis, J.H., 2006. The influence of soft tissue movement on ground reaction forces, joint torques and joint reaction forces in drop landings. Journal of Biomechanics 39, 119-124.

Pandy, M.G., Zajac, F.E., Sim, E., Levine, W.S., 1990. An optimal control model for maximum height human jumping. Journal of Biomechanics 23, 1185-1198.

Pandy, M.G., Zajac, F.E., 1991. Optimal muscular coordination strategies for jumping. Journal of Buiomechanics 24, 1-10. 
Pierrynowski, M.R., 1995. Analytical representation of muscle line of action and geometry. In Allard, P., Stokes, I.A.F. and Blanchi, J. (Eds.), Three-dimensional analysis of human movement. Champaign: Human Kinetics.

Soest, A.J. van, Bobbert, M.F., Ingen Schenau, G.J. van, 1994. A control strategy for the execution of explosive movements from varying starting positions. Journal of Neurophysiology 71, 1390-1402.

Soest, A.J. van, Schwab, A.L., Bobbert, M.F., Ingen Schenau, G.J. van, 1993. The influence of the biarticularity of the gastrocnemius muscle on vertical jumping achievement. Journal of Biomechanics 26, 1-8.

Sprigings, E., Miller, D. I., 2004. Optimal knee extension timing in springboard and platform dives from the reverse group. Journal of Applied Biomechanics 20, 275-290.

Thelen, D.G., Anderson, F.C., Delp, S.L., 2003. Generating dynamic simulations of movement using computed muscle control. Journal of Biomechanics 36, 321-328.

Westing, S.H., Cresswell, A.G., Thorstensson, A., 1991. Muscle activation during maximal voluntary eccentric and concentric knee extension. European Journal of Applied Physiology 62, 104-108.

Westing, S.H., Seger, J.Y. and Thorstensson, A., 1990. Effects of electrical stimulation on eccentric and concentric torque-velocity relationships during knee extension in man. Acta Physiologica Scandinavica 140, 17-22.

Wilson, C., 2003. Optimisation of performance in running jumps. Doctoral dissertation, Loughborough University, UK.

Wilson, C., King, M.A., Yeadon, M.R., 2006. Determination of subject-specific model parameters for visco-elastic elements. Journal of Biomechanics 39, 1883-1890.

Wood, G.A., Jennings, L.S., 1979. On the use of spline functions for data smoothing. Journal of Biomechanics 12, 447-479.

Yeadon, M.R., 1990a. The simulation of aerial movement - I: The determination of orientation angles from film data. Journal of Biomechanics, 23, 59-66.

Yeadon, M.R., 1990b. The simulation of aerial movement - II: A mathematical inertia model of the human body. Journal of Biomechanics, 23, 67-74.

Yeadon, M.R., 1990c. The simulation of aerial movement - III: The determination of the angular momentum of the human body. Journal of Biomechanics 23, 75-83.

Yeadon, M.R., Atha, J., Hales, F.D., 1990. The simulation of aerial movement - IV: A computer simulation model. Journal of Biomechanics 23, 85-89.

Yeadon, M.R., Challis J.H., 1994. The future of performance-related sports biomechanics research. Journal of Sports Sciences 12, 3-32.

Yeadon, M.R., Hiley, M.J., 2000. The mechanics of the backward giant circle on the high bar. Human Movement Science 19, 153-173.

Yeadon, M.R., King, M.A., 2002. Evaluation of a torque-driven simulation model of tumbling. Journal of Applied Biomechanics 18, 195-206.

Yeadon, M.R., King, M.A., Wilson, C., 2006. Modelling the maximum voluntary joint torque/angular velocity relationship in human movement. Journal of Biomechanics 39, 476-482. 